Modelo econométrico para el análisis de la elección de modo de transporte en viajes domésticos: el rol de las variables latentes

Claudia Muñoz, Beatriz Elena Pineda Uribe y Margarita Rosa Gómez 
Claudia Muñoz, Beatriz. Elena Pineda Uribe y Margarita Rosa Gómez.

Modelo econométrico para el análisis de la elección de modo de transporte en viajes domésticos: el rol de las variables latentes

Resumen: Las percepciones de seguridad y comodidad relacionadas con viajar en bus o en avión pueden afectar las decisiones de elección de los pasajeros en viajes domésticos. En este artículo se incorporaron las variables latentes seguridady comodidad dentro de los modelos econométricos para estudiar la elección del modo de transporte para pasajeros que viajan en el trayecto Medellin-Barranquilla. En el modelo calibrado se incluyeron variables asociadas al modo de transporte, como el costo, el tiempo de viaje y las frecuencias, además de las variables de percepción. El modelo de elección discreta con variables latentes (VLs) se estimó secuencialmente: en primer lugar, se obtuvieron los parámetros del modelo de múltiples indicadores múltiples causas (MIMIC) y — posteriormente - se calibró el modelo bíbrido de elección. El modelo calibrado permitió identificar las variables que inciden en la elección del modo de transporte aéreo o terrestre. Los resultados obtenidos son una herramienta eficaz en la toma de decisiones para las empresas que operan en cada uno de los modos.

Palabras clave: demanda de transporte, modelos econométricos, transporte aéreo.

Clasificación JEL: R15, R41, L93.

Econometric model for the analysis of the transportation mode choice in domestic trips: the role of latent variables

Abstract: Safety and comfort perceptions related to travel by bus or plane could affect passenger choice behavior in domestic trips. In this research, the safety and comfort latent variables were incorporated into the econometric models to study the choice of transportation mode for passengers who travel along the Medellin - Barranquilla route. Variables associated with the transportation mode such as cost, travel time, and frequency were included in the calibrated model and perceptional variables. The discrete choice model with latent variables ( $L V s$ ) found in this research was sequentially estimated. Firstly, the parameters of Multiple Indicators Multiple Causes Model (MIMIC) were found, and then the bybrid choice model was calibrated. The calibrated model allowed us to identify the variables that affect the decision-making process for air and land transportation mode choice. The results are an effective decision-making tool for companies that operate in each of the transportation modes.

Keywords: air transportation, econometric models, transportation demand.

https://doi.org/10.17533/udea.le.n96a345079

\section{(c) BY-NC-SA}

Este artículo y sus anexos se distribuyen por la revista Lecturas de Economía bajo los términos de la Licencia Creative Commons Atribución-NoComercial-CompartirIgual 4.0. https://creativecommons.org/licenses/by-nc-sa/4.0/ 
Modèle économétrique pour l'analyse du choix de mode de transport dans les voyages intérieurs: le rôle des variables latentes

Résumé: Les perceptions de sécurité et de confort liées aux voyages en autobus ou en avion peuvent influer sur les décisions et choix des passagers en matière de voyages intérieurs. Dans cet article, les variables latentes de sécurité et de confort ont été incorporées aux modèles économétriques pour étudier le choix du mode de transport pour les passagers voyageant sur le trajet Medellin-Barranquilla. Le modèle calibré comprenait des variables associées au mode de transport, comme le coût, le temps de déplacement et les fréquences, en plus des variables de perception. Le modèle à choix discret avec variables latentes (VLs) a été estimé séquentiellement: d'abord, les paramètres du modèle à causes multiples e à indicateurs multiples (MIMIC) ont été obtenus et, par la suite, le modèle hybride de choix a été étalonné. Le modèle calibré a permis d'identifier les variables qui influent sur le choix du mode de transport aérien ou terrestre. Les résultats obtenus sont un outil de prise de décision efficace pour les entreprises opérant dans chacun des modes.

Mots clés: demande de transport, modèles économétriques, transport aérien

Cómo citar / How to cite this item:

Muñoz, C., Pineda Uribe, B. E., \& Gómez, M. R. (2022). Modelo econométrico para el análisis de la elección de modo de transporte en viajes domésticos: el rol de las variables latentes. Lecturas de Economía, 96, 145-169. https://doi.org/10.17533/udea.le.n96a345079 


\title{
Modelo econométrico para el análisis de la elección de modo de transporte en viajes domésticos: el rol de las variables latentes
}

\author{
Claudia Muñoz $\circledast^{\mathrm{a}}$, Beatriz Elena Pineda Uribe $\oplus^{\mathrm{b}}$ y Margarita Rosa \\ Gómez $\oplus^{c}$
}

-Introducción. -I. Marco teórico y revisión de literatura. -II. Metodología. -III. Resultados y discusión. -Conclusiones. -Agradecimientos. -Referencias.

Primera versión recibida el 28 de enero de 2021; versión final aceptada el 04 de septiembre de 2021

\section{Introducción}

En algunos países las aerolíneas de bajo costo han demostrado tener una ventaja competitiva sobre otras, debido a sus tarifas más bajas y niveles similares de calidad de servicio (Hyun et al., 2011). Pero no solo compiten con otras aerolíneas, sino también con otros modos de transporte como el terrestre donde en el territorio colombiano tiene gran protagonismo.

El Ministerio de Transporte ha emitido reportes en los que demuestra que el modo de transporte interdepartamental de pasajeros más utilizado es el terrestre. Ahora bien, este panorama ha tenido un cambio significativo debido a la incursión de aerolíneas de bajo costo (low-cost) en el mercado colombiano, ocasionando una disminución en las tarifas aéreas respecto a años anteriores.

En Colombia, la incorporación de aerolíneas low-cost — como Viva Air en 2012 y Wingo en 2016 - permitió el surgimiento de la competencia directa

a Clandia Muñoz: profesora asistente de la Universidad de Antioquia, Facultad de Ingeniería, Medellín, Colombia. Profesora cátedra Universidad Nacional de Colombia, Facultad de Minas, Medellín, Colombia. Dirección electrónica: claudia.munoz1@udea.edu.co https:/ / orcid.org/0000-0003-4961-5308

b Beatriz Elena Pineda Uribe: profesora de tiempo completo del Politécnico Colombiano Jaime Isaza Cadavid, Facultad de Ingeniería, Medellín, Colombia. Dirección electrónica: beatrizpineda@elpoli.edu.co https://orcid.org/0000-0003-2467-0765

c Margarita Rosa Gómez: profesora de cátedra de la Universidad Nacional de Colombia, Facultad de Minas, Medellín, Colombia. Dirección electrónica: mrgomezbet@unal.edu.co https://orcid.org/0000-0003-0924-7184 
Muñoz, Pineda Uribe y Gómez: Modelo econométrico para el análisis de la elección de modo...

entre el transporte aéreo y el transporte terrestre. En 2016 el transporte aéreo presentó un crecimiento de $45 \%$ respecto al año 2012. En solo cuatro años pasó de 24,7 millones de pasajeros a 35,8 millones de pasajeros en el 2016 (Aeronáutica Civil de Colombia, 2017). Así, la facilidad de encontrar mejores ofertas en tiquetes aéreos ha hecho que más pasajeros tengan la posibilidad de transportarse por este medio, encontrando — incluso — tarifas en algunas ocasiones menores, produciendo con esto un traslado de pasajeros cautivos por el transporte terrestre como potenciales del transporte aéreo.

Con base en las estadísticas reportadas por la Aerocivil (Aeronáutica Civil de Colombia, 2017) —específicamente para el trayecto MedellínBarranquilla-, la cantidad de personas movilizadas en el modo aéreo durante 2017 fue de 175382 pasajeros, mientras que en el transporte terrestre fue de 25178. Dada la importancia de estos dos medios de transporte y la demanda actual que tiene cada uno de ellos, es interesante estudiar el comportamiento de los usuarios teniendo en cuenta que estos eligen la alternativa más atractiva sujetos a ciertas restricciones (McFadden \& Domencich, 1975). De esta manera, se puede realizar la predicción de la demanda futura de pasajeros y la respectiva generación de estrategias de distribución.

Los servicios de transporte surgen como consecuencia de la necesidad que tienen los individuos de realizar actividades que implican desplazamientos. El objetivo de un sistema de transporte es satisfacer la demanda mediante la provisión de una oferta adecuada que corresponda a sus exigencias. La pregunta que se hacen los administradores de las empresas de transporte terrestre y aéreo de pasajeros es: ¿Cómo satisfacer la demanda de viajes a diferentes horas del día en cada uno de los trayectos interdepartamentales, restringidos por una operación limitada? La respuesta a esto depende - en gran medida- de las estimaciones que se realicen de esta demanda.

La demanda de transporte depende de las variables socioeconómicas que caracterizan a los usuarios y de los atributos de las alternativas — que son analizadas por el individuo al momento de tomar la decisión-, destacando entre ellas el tiempo de viaje y el costo del tiquete, además de variables latentes de percepción como la seguridad y la comodidad que ofrece cada sistema de transporte. 
Los modelos de demanda desagregados constituyen actualmente, una herramienta de análisis eficaz para abordar el problema del estudio de la demanda de transporte (Espino et al, 2004; Ortúzar \& Willumsen, 2011;), por lo tanto, por medio de los modelos de elección discreta se puede analizar y valorar la elección de una alternativa dentro de un grupo exclusivo y particular de opciones.

Este documento se encuentra estructurado de la siguiente forma. Posterior a la introducción, se presenta el marco teórico y la revisión de la literatura relacionada con los modelos de elección discreta. En la siguiente sección se detalla la metodología para evaluar las elecciones de modo de transporte para viajes domésticos. En la penúltima sección se analiza los resultados de los modelos calibrados y su respectiva discusión. Finalmente, se presentan las conclusiones.

\section{Marco teórico y revisión de la literatura}

\section{A. Modelos clásicos de elección discreta}

En estos, se relaciona la probabilidad condicional de una elección con una serie de factores explicativos, que recogen las características de los individuos que toman las decisiones —así como los atributos de las alternativas - y permiten calcular la probabilidad de que un individuo con ciertas características realice una elección dentro de un conjunto de alternativas — por ejemplo, utilizar el transporte terrestre o aéreo- con el fin de realizar un desplazamiento (Drabas \& Wu, 2013).

La función de utilidad para cada alternativa a evaluar, está representada por la ecuación 1:

$$
U_{i q}=V_{i q}+\varepsilon_{i q}
$$

Así la utilidad de la alternativa $i$ para un individuo $q$, es $U_{i q}$, está representada por la suma de un término conocido por el modelador y otro aleatorio (McFadden \& Domencich, 1975). $V_{i q}$ corresponde a la parte medible de la utilidad, que es función de los atributos de la alternativa y las características del individuo y $\varepsilon_{i q}$ es un término de error estocástico 
Muñoz, Pineda Uribe y Gómez: Modelo econométrico para el análisis de la elección de modo...

que incluye todos los factores desconocidos o no tenidos en cuenta por el modelador.

La teoría de la utilidad aleatoria considera que el individuo $q$ escoge la alternativa de máxima utilidad, esto es, escoge la opción $i$ si (ver ecuación 2):

$$
U_{i q} \geq U_{j q}
$$

donde se origina la desigualdad de la ecuación 3:

$$
V_{j q}-V_{i q} \geq \varepsilon_{i q}-\varepsilon_{j q}
$$

De esta forma, la probabilidad de escoger la alternativa $A_{i}$ es dada por la ecuación 4:

$$
P_{i q}=\operatorname{Pr}\left\{\varepsilon_{j q} \leq \varepsilon_{i q}+V_{i q}-V_{j q}, \forall A_{j} \varepsilon A_{q}\right\} .
$$

Sin duda, el modelo más utilizado es el logit multinomial (MNL) y surge al asumir que los términos $\varepsilon_{i q}$ son independientes e idénticamente distribuidos Gumbell $\left(V_{j q}, \lambda\right)$. El término $\lambda$ está asociado a la varianza de los errores, siendo un parámetro de escala del modelo.

La probabilidad de que la alternativa $i$, sea elegida por el individuo $q$; está dada por la ecuación 5:

$$
P_{i q}=\frac{e^{\beta \sum_{k} \theta_{k} X_{i k q}}}{\sum_{j} e^{\beta \sum_{k} \theta_{k} X j k q}} .
$$

La estimación del modelo consiste en encontrar los coeficientes $\theta_{k}$ que generan más a menudo la muestra observada, es decir los más verosímiles, que son aquellos que maximizan la probabilidad de que ocurra un suceso observado.

Los modelos clásicos de elección discreta pierden capacidad explicativa al tomar en cuenta solo los atributos y características observables de los modos de transporte e individuos, porque no logran modelar la heterogeneidad de los individuos, la cual afecta considerablemente la elección modal. Para mejorar esta debilidad de los modelos clásicos, En la investigación de Ben-Akiva et al. (2002a) se presentó un nuevo marco de trabajo sobre los modelos de elección discreta, incluyendo en ellos variables adicionales de comportamiento. 


\section{B. Modelos híbridos de elección}

Las preferencias se pueden estudiar a través de encuestas de preferencias declaradas, donde al individuo se le presentan alternativas reales o hipotéticas y este elige de acuerdo con los atributos de las alternativas (Morikawa et al., 2002). En los modelos híbridos se utilizan indicadores de percepción para la determinación de las variables latentes (VLs). Estos indicadores son mediciones cuantitativas que se hacen directamente a los individuos. Posteriormente, con las VLs, más las variables explicativas, se calibra un modelo de elección discreta estándar representado en la Figura 1.

Figura 1. Modelo integrado de elección y variables latentes

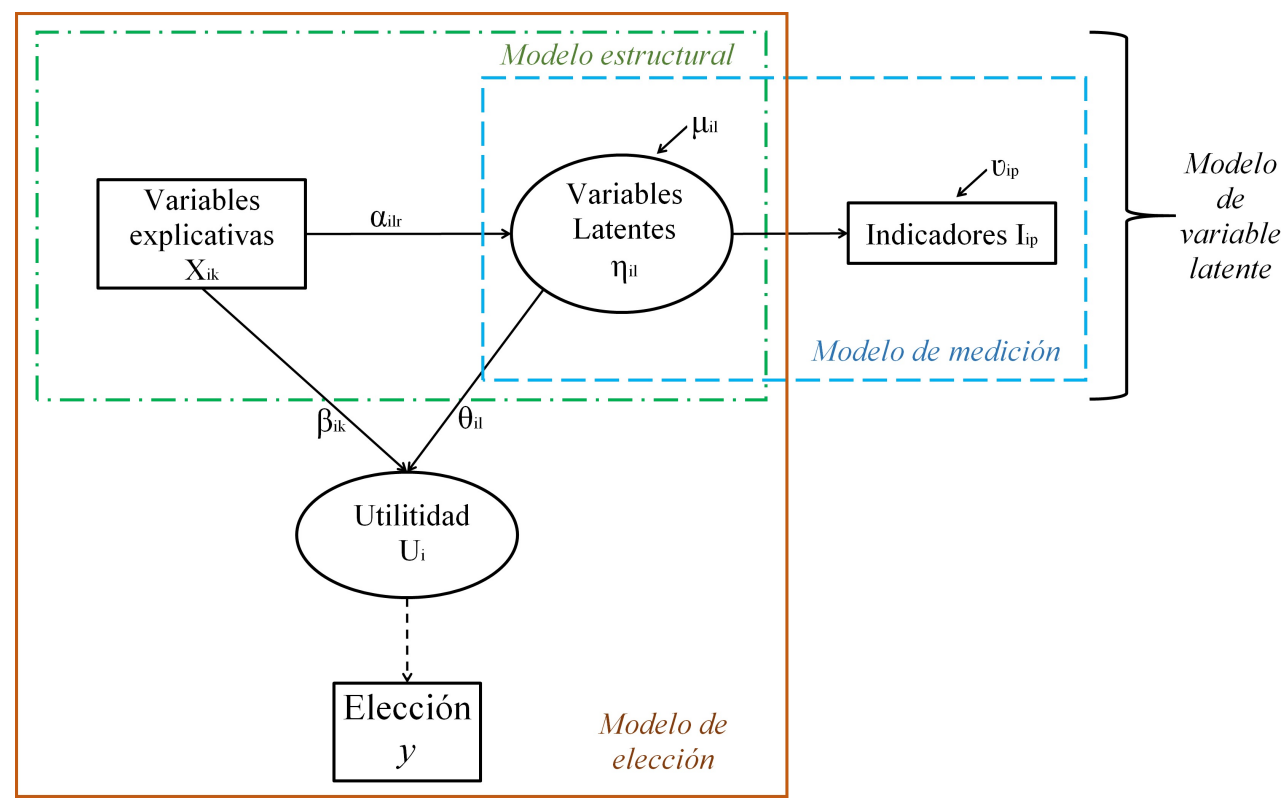

Fuente: elaboración propia basado en Ben-Akiva et al. (2002a).

Este enfoque constituye una metodología secuencial, ya que — previo a estimar el modelo de elección - es necesario procesar los datos cuantitativos entregados por los indicadores para transformarlos en VLs. Este primer paso, conocido como modelo de VL (Bolduc et al., 2003), se adapta a un modelo 
Muñoz, Pineda Uribe y Gómez: Modelo econométrico para el análisis de la elección de modo...

MIMIC (Johansson et al., 2005) donde, a través de la aplicación del análisis factorial, es posible determinar las VLs y sus respectivos valores en el sistema de ecuaciones. En el modelo de variables latentes, $\eta_{i l q}$ representa un vector de VLs que en el caso de este artículo serán las variables seguridad y comodidad, $\alpha_{i l r}$ son parámetros a estimar, $X_{i k}$ es un conjunto de variables observadas — variables socioeconómicas, características del modo, etcétera-.

Las ecuaciones estructurales del modelo MIMIC son representadas como:

$$
\eta_{i l q}=\sum_{r} \alpha_{i l r} X_{i r q}+\mu_{i l q}
$$

Por otra parte, el modelo de medición contiene una ecuación por cada indicador $p$, como se muestra en la ecuación 7. Por lo tanto, el modelo de medición mide la relación entre las VLs $\left(\eta_{i l q}\right)$ y los indicadores $I_{i p q}$

$$
I_{i p q}=\sum_{l} \gamma_{i l p} \eta_{i l q}+\nu_{i p q}
$$

donde $\gamma_{i l p}$ son parametros a ser estimados. $\mu_{i l q}$ y $\nu_{i p q}$ son términos de error. Las ecuaciones 6 y 7 deben ser consideradas a la vez en la estimación de parámetros con el objetivo de conocer el término $\eta_{i l q}$ (Bollen, 1989).

Si las VLs $\left(\eta_{i l q}\right)$ son incluidas, la utilidad sistemática de la ecuación 1 puede ser reformulada para acomodar los elementos del modelo híbrido, donde $\beta_{i k}$ y $\theta_{i l}$ son los parámetros por estimar y están asociados - respectivamente- con los atributos tangibles y con las VLs, como se presenta en la ecuación 8:

$$
V_{i q}=\sum_{k} \beta_{i k} X_{i k q}+\sum_{l} \theta_{i l} \eta_{i l q} .
$$

La inclusión de las VLs se ha dado con éxito en la modelación en los diferentes modos de transporte. Las VLs comúnmente introducidas en los modelos de demanda de transporte han sido la seguridad, la comodidad entre otras (Johansson et al., 2005; Johansson et al. (2005); Kitrinou et al., 2010). Problemas de ilación. Se recomienda ampliar o 
conectar de manera coherente con el párrafo anterior. Se modifica estructura del párrafoEn el caso del transporte aéreo de pasajeros, los trabajos de Tam et al. (2011) y Fleischer et al. (2012) demostraron que la inclusión de las variables calidad del servicio y miedo a volar respectivamente son consideradas estadísticamente significativas en el proceso de elección. A su vez, Yazdanpanah \& Hosseinlou (2016) y Kim et al. (2017) incorporaron las variables personalidad y satisfacción dentro de los modelos de elección de modo terrestre de transporte.

El objetivo de esta investigación es encontrar un modelo híbrido de elección de modo de transporte para el trayecto Medellín-Barranquilla, basado en los resultados de una encuesta de preferencias declaradas realizadas para los modos terrestre y aéreo. Los modelos econométricos encontrados permiten explicar y cuantificar la influencia que las VLs seguridad y comodidad tiene en la decisión tomada por el usuario y que ayudan a evaluar posibles políticas de distribución de pasajeros para las empresas que operan en los modos terrestre y aéreo.

\section{Metodología}

El modelo propuesto para evaluar las elecciones de modo de transporte para viajes domésticos consideró una de las rutas que mayor movimiento de pasajeros tiene en el territorio colombiano. El trayecto Medellín-Barranquilla fue elegido para el desarrollo del estudio, por ser Barranquilla una de las ciudades más distantes desde la capital Antioqueña, y donde existe una competencia directa entre el transporte terrestre y aéreo de pasajeros. La Figura 2 presenta la cantidad de pasajeros que se movilizaron por cada uno de estos dos modos en cada año y se aprecia claramente el notable crecimiento del transporte aéreo entre los años 2011 y 2016.

El proceso usado para obtener la representatividad de la población en la muestra fue basado en la expresión de Cochran (1977), quien desarrolló una expresión para calcular el tamaño de la muestra para una población infinita:

$$
n=\frac{z^{2} p q}{e^{2}}
$$


Muñoz, Pineda Uribe y Gómez: Modelo econométrico para el análisis de la elección de modo...

donde $n$ es el tamaño de la muestra, $p$ es la proporción estimada de un atributo presente en la población, $q$ es calculado como $1-p$ y $z$ representa el $z$-valor de la distribución normal para $\alpha / 2$, donde $(1-\alpha)$ x $100 \%$ es el nivel de confianza. En este artículo, la población es asumida como infinita, donde para el caso extremo, p y q son iguales a 0,5 y se tiene un nivel de confianza del con un $\pm 5 \%$ de precisión. Así, el tamaño de la muestra $n$ debe ser mínimo 384 . En esta investigación, se llevaron a cabo 420 encuestas $(n+36)$, teniendo en cuenta la probabilidad de datos inconsistentes.

Figura 2. Movimiento de pasajeros Medellin-Barranquilla 2011-2017

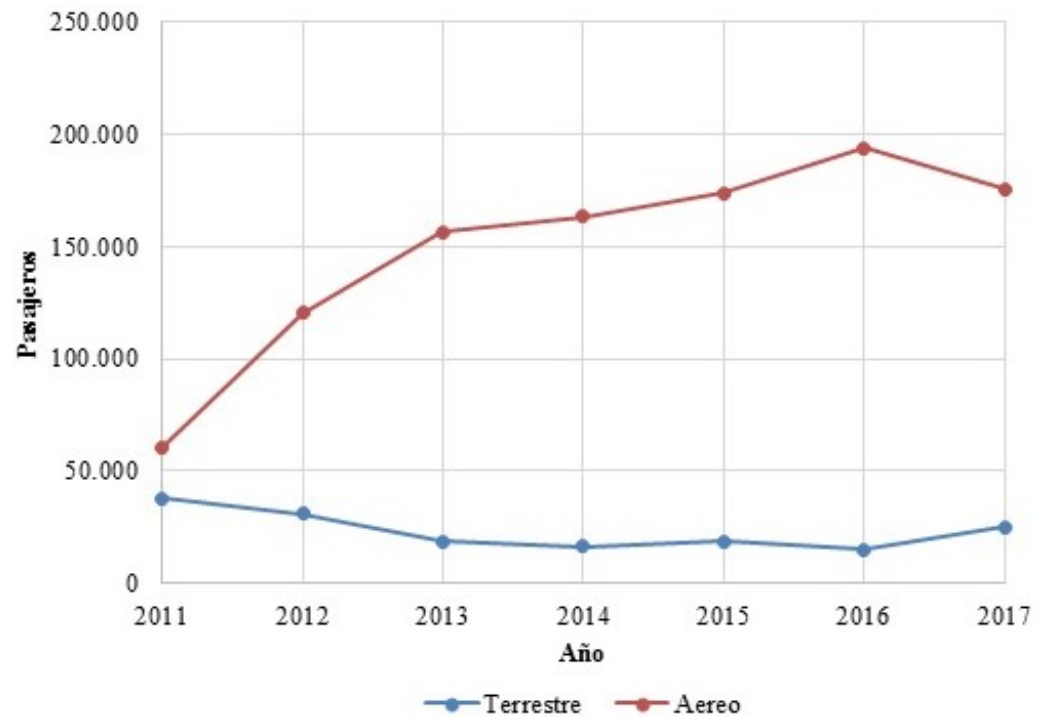

Fuente: elaboración propia con base en información de Terminales de Transporte Medellín S. A. (2018) y Aeronáutica Civil de Colombia (2017).

Con el objetivo de extraer una muestra representativa de todos los pasajeros y reflejar el patrón de uso real del modo aéreo y terrestre para el trayecto Medellín-Barranquilla, fue necesario un muestreo de cuotas para la encuesta. La Tabla 1 está basada en las frecuencias relativas de la participación del mercado de los modos aéreo y terrestre en el 2017. El total de la muestra se estratificó por tamaño en cada categoría. Por lo tanto, los datos empleados 
en este artículo son representativos de la población de pasajeros en el trayecto Medellín-Barranquilla.

Tabla 1. Tasa de muestreo basada en las cuotas del mercado

\begin{tabular}{cccc}
\hline \multirow{2}{*}{ Modo } & \multicolumn{2}{c}{ Cuota del mercado en 2017} & \multirow{2}{*}{ Muestra recolectada } \\
\cline { 2 - 3 } & Población & Frecuencia relativa & \\
\hline Aéreo & 175385 & $87 \%$ & 360 \\
Terrestre & 25178 & $13 \%$ & 60 \\
Total & 200563 & $100 \%$ & 420 \\
\hline
\end{tabular}

Fuente: elaboración propia.

Para la obtención del modelo de elección discreta se realizaron encuestas de preferencias declaradas. Estas se realizaron cara a cara en el año 2018, debido a que este método de muestreo ofrece mejores resultados en términos de representatividad (Szolnoki \& Hoffmann, 2013). Los datos se recopilaron en las puertas de embarque de las terminales aérea y terrestre, donde los pasajeros completaban el cuestionario. Los escenarios de elección fueron presentados a usuarios de los dos modos de transporte evaluados en el trayecto Medellín-Barranquilla.

Los atributos usados en el estudio fueron el costo del tiquete, el tiempo de viaje y las frecuencias. En la Tabla 2 se presentan los atributos y niveles usados en el experimento de elección, los cuales fueron determinados con base en los valores de las operaciones actuales de los diferentes modos de transporte.

\section{A. Diseño de la encuesta de preferencias declaradas (PD)}

Un modelo de demanda de transporte requiere recopilar una amplia variedad de información, derivada de diferentes fuentes, como son las encuestas (Żochowska et al., 2017). Las encuestas de preferencias declaradas —PD_corresponden a un enfoque ampliamente aplicado por los investigadores para comprender los comportamientos de elección de los viajeros. La encuesta PD se basa en perfiles hipotéticos, diseñados para evaluar las preferencias de los usuarios por atributos específicos. Dada la selección de atributos y sus niveles asociados, se realizó un experimento PD utilizando un diseño ortogonal 
Muñoz, Pineda Uribe y Gómez: Modelo econométrico para el análisis de la elección de modo...

Tabla 2. Atributos y niveles

\begin{tabular}{lccc}
\hline \multirow{2}{*}{ Atributo } & Niveles & \multicolumn{2}{c}{ Alternativas (modos de transporte) } \\
\cline { 3 - 4 } & & Terrestre & Aéreo \\
\hline Costo del tiquete* & 0 & 80000 & 160000 \\
& 1 & 100000 & 200000 \\
Tiempo de viaje & 2 & 120000 & 240000 \\
& 0 & 12 & 2 \\
Frecuencias & 1 & 14 & 6 \\
& 2 & 16 & 6 frecuencias/día \\
& 0 & 10 frecuencias/día & 7 frecuencias/día \\
& 1 & 12 frecuencias/día & 9 frecuencias/día \\
\hline
\end{tabular}

* costo del tiquete de un solo trayecto

Fuente: elaboración propia.

completo, donde el número de situaciones de elección que componen diseño factorial es $L^{M A 1}$ o diseño ortogonal (Hensheret al., 2005). Este enfoque ha sido ampliamente utilizado en estudios de transporte por Márquez et al. (2019), Tsai y Chen (2019), Lee y Yip (2017), Wen et al. (2019), Yang et al. (2014) y Fleischer et al. (2012).

El diseño factorial ortogonal permite que todos los atributos no estén correlacionados y que sus niveles de variación estén equilibrados. Sin embargo, en los últimos años se ha utilizado el método de diseño eficiente para minimizar los errores estándar. Una desventaja del diseño eficiente es la necesidad de conocer previamente los parámetros de los atributos a evaluar, esto hace que el diseño experimental sea sensible a una especificación incorrecta de los parámetros anteriores. Por lo tanto, la aplicación del enfoque del diseño factorial ortogonal refleja nuestra preferencia por la independencia estadística sobre la eficiencia. Si se aplicara un diseño factorial completo para las dos alternativas — modos de transporte-, con sus tres atributos

1 Nótese que $L$ es el número de niveles de atributo, $\mathrm{M}$ el número de alternativas y $\mathrm{A}$ el número de atributos. 
y sus tres niveles de variación, se produciría $3^{2 \times 3}$ posibles combinaciones. Dada la cantidad de combinaciones, se aplicó un diseño factorial ortogonal utilizando el software NGENE con el objetivo de reducir las combinaciones a un tamaño manejable de nueve escenarios.

Antes de la aplicación de encuesta definitiva se realizó una prueba piloto a 60 individuos, con el objetivo detectar problemas potenciales con respecto a factores como la longitud del cuestionario, la fatiga de los encuestados y la claridad de la encuesta.

Dentro de la encuesta se plantearon diferentes escenarios de elección y cada uno de los modos fue representado en términos del costo del tiquete, del tiempo de viaje y de las frecuencias. Cada encuestado debía evaluar nueve escenarios y elegir una de las dos posibles alternativas. En el desarrollo de la encuesta se incluyeron variables socioeconómicas como género, edad, ocupación, número de viajes realizados por año, motivo del viaje, ingresos, estrato socioeconómico, número de acompañantes, número de maletas.

Tabla 3. Indicadores de las VLs

\begin{tabular}{ll}
\hline Variable latente & Indicadores \\
\hline Seguridad (S) & $\begin{array}{l}\text { Seguridad en cuanto a robo o pérdida de objetos en el medio de } \\
\text { transporte (SR) } \\
\text { Seguridad en cuanto a entrega de equipaje en buen estado (SE) }\end{array}$ \\
\hline & $\begin{array}{l}\text { Comodidad en cuanto a la facilidad para acceder al modo de transporte } \\
\text { desde el origen (CCF) }\end{array}$ \\
Comodidad (C) & $\begin{array}{l}\text { Comodidad en cuanto a la suavidad en el desplazamiento en un viaje } \\
\text { (CSD) } \\
\text { Comodidad en cuanto a las sillas (CS) }\end{array}$ \\
\hline
\end{tabular}

Fuente: elaboración propia.

Las VLs seguridad y comodidad fueron evaluadas para cada modo a través de los indicadores presentados en la Tabla 3, los cuales fueron calificados en una escala tipo Likert de 1 a 5, siendo 1 "muy inseguro" o "muy incómodo", y 5 "muy seguro" o "muy cómodo". Los indicadores para las variables latentes seguridad y comodidad evaluados para los modos aéreo y terrestre 
Muñoz, Pineda Uribe y Gómez: Modelo econométrico para el análisis de la elección de modo...

fueron basados en investigaciones previas desarrolladas para viajes urbanos (Córdoba, 2011; Márquez, 2016).

\section{B. Descripción de la muestra}

Fueron encuestados 420 pasajeros, quienes realizaron un viaje de Medellín a Barranquilla en el modo aéreo o terrestre. Para la muestra se encontró que el $16 \%$ de los encuestados tienen ingresos mensuales entre USD 500 y USD 1000, el $38 \%$ entre USD 1000 y USD 1500, el $20 \%$ entre USD 1500 y USD 2000 y el $14 \%$ mayor a USD 2000 y en un menor porcentaje (12\%) los individuos que poseen ingresos menores a USD 500. Respecto al motivo del viaje, el $72 \%$ de los individuos se movilizaron por trabajo o negocios, lo cual puede estar relacionado con el hecho de que las encuestas no fueron realizadas en temporada de vacaciones. La muestra estuvo distribuida uniformemente entre mujeres y hombres, representando el $48 \%$ y $52 \%$, respectivamente.

\section{Calibración del modelo}

En la fase de calibración, inicialmente se encontraron modelos logit multinomial. Posteriormente, se utilizó el método secuencial, empleado para estimar modelos en dos etapas (Ben-Akiva et al., 2002b). Esta etapa de la investigación tuvo varios procesos: primero, se obtuvieron los modelos MIMIC; estos modelos permiten observar las variables latentes a través de unos indicadores y explicarlas a través de variables relacionadas con factores socioeconómicos del usuario. Los modelos encontrados permitieron analizar el comportamiento de los individuos ante las dos alternativas existentes: transporte terrestre o aéreo para el trayecto Medellín-Barranquilla.

\section{Resultados y discusión}

Para comenzar se realizó la modelación de las VLs seguridad y comodidad, luego se calibraron los modelos con los datos de las PD, con el objeto de encontrar las variables significativas y, por último, con la función de utilidad obtenida se calcularon las cuotas del mercado de cada uno de los modos incluidos en el estudio. 
Las variables contenidas en la PD fueron: el tiempo de viaje (Tv), el costo de viaje $(\mathrm{C})$ y las frecuencias ( $\mathrm{Fr}$ ); con base en la teoría microeconómica, los signos esperados para $\mathrm{Tv}$ y $\mathrm{C}$ son negativos y para Fr es positivo.

Diferentes especificaciones de modelos fueron probadas. Estos modelos difieren en el número de variables estimadas. Además, en cada modelo se realizó un análisis de correlación entre las variables, asegurando con esto que todas las variables que resultaban significativas no estuvieran correlacionadas.

\section{A. Modelos de múltiples indicadores múltiples causas (MIMIC)}

En las tablas 4 y 5 se presentan los resultados de los parámetros de las variables explicativas y de los indicadores de las VLs seguridad y comodidad que resultan del modelo MIMIC.

Tabla 4. Modelo MIMIC para la VL seguridad

\begin{tabular}{ccccc}
\hline $\begin{array}{c}\text { Variables Exógenas e } \\
\text { indicadores }\end{array}$ & \multicolumn{2}{c}{$\begin{array}{c}\text { VL seguridad modo } \\
\text { terrestre (SEGT) }\end{array}$} & \multicolumn{2}{c}{$\begin{array}{c}\text { VL seguridad modo } \\
\text { aéreo (SEGA) }\end{array}$} \\
\cline { 2 - 5 } & Parámetro & Test-t & Parámetro & Test-t \\
\hline Edad (ED) & 0,368 & 2,276 & 0,478 & 3,014 \\
Género (GEN) & 0,234 & 2,057 & 0,567 & 2,284 \\
Indicador SR & $1,000^{*}$ & & 0,792 & 3,851 \\
Indicador SE & 0,864 & 2,342 & $1,000^{*}$ & \\
\hline
\end{tabular}

${ }^{*}$ No se probó la significancia porque estos indicadores se fijan en 1,0 para fijar la escala de las variables latentes

Fuente: elaboración propia.

Como se muestra en la Tabla 4, la VL seguridad fue evaluada para el modo terrestre (SEGT) y para el aéreo (SEGA). De igual manera la VL comodidad se evaluó para el modo terrestre (COMT) y aéreo (COMA) y los resultados del modelo se presentan en la Tabla 5. Como se observa todos los parámetros son significativamente diferentes de cero con un nivel de significancia del $5 \%$.

Las tablas 4 y 5 muestran que el género y la edad permiten explicar las VLs del estudio. En términos de género, las mujeres encuestadas consideran 
Muñoz, Pineda Uribe y Gómez: Modelo econométrico para el análisis de la elección de modo...

más seguro el transporte aéreo que el terrestre. Por otro lado, a mayor edad se tiene una percepción mayor de comodidad relacionada con viajar en el modo aéreo.

Tabla 5. Modelo MIMIC para la VL comodidad

\begin{tabular}{ccccc}
\hline $\begin{array}{c}\text { Variables Exógenas e } \\
\text { indicadores }\end{array}$ & \multicolumn{2}{c}{$\begin{array}{c}\text { VL Comodidad modo } \\
\text { terrestre (COMT) }\end{array}$} & \multicolumn{2}{c}{$\begin{array}{c}\text { VL Comodidad modo } \\
\text { aéreo (COMA) }\end{array}$} \\
\cline { 2 - 5 } & Parámetro & Test-t & Parámetro & Test-t \\
\hline Edad (ED) & 0,433 & 2,102 & 0,833 & 2,482 \\
Género (GEN) & 0,721 & 1,969 & 0,521 & 1,969 \\
Indicador CCF & 0,884 & 2,872 & 0,802 & 2,207 \\
Indicador CSD & $1,000^{*}$ & & $1,000^{*}$ & \\
Indicador CS & 0,752 & 2,922 & 0,928 & 2,234 \\
\hline
\end{tabular}

* No se probó la significancia porque estos indicadores se fijan en 1,0 para fijar la escala de las variables latentes

Fuente: elaboración propia

\section{B. Resultados de los modelos de elección discreta (MED)}

En el proceso de calibración de los modelos —inicialmente a partir de los modelos MIMIC - se calcularon los valores esperados de las VLs para cada viajero. La Tabla 6 presenta los resultados de un modelo logit multinomial (MNL) sin VLs, así como el modelo híbrido, el cual incluye las variables de percepción de seguridad y comodidad. Para cada parámetro el valor de la prueba t se realiza cambio se presenta entre paréntesis. Ambos modelos consideran el efecto de panel que se produce al tener nueve observaciones diferentes en las PD por individuo (Cantillo et al., 2007).

El modelo MNL es la especificación más simple, ya que no incluye el efecto de la VLs seguridad y comodidad. Como se presenta en la Tabla 6, todos los parámetros son significativamente diferentes de cero con un nivel de significancia del $5 \%$. Los signos de los parámetros corresponden al supuesto subyacente de la teoría microeconómica. Por lo tanto, cuando aumentan los 
tiempos de viaje y las tarifas, las utilidades de los modos terrestre y aéreo se reducen. Ambos modelos muestran que los pasajeros de la ruta MedellínBarranquilla perciben que el tiempo de viaje es un atributo más significativo que la tarifa. En general los viajeros prefieren tarifas más bajas, viajes más cortos y mayor número de frecuencias.

Tabla 6. Resultados del modelo de elección

\begin{tabular}{lcc}
\hline Variable & ParámetrosModelo sin VLs & ParámetrosModelo híbrido \\
\hline Constante específica & $1,216(3,873)$ & $0,606(2,461)$ \\
Tiempo de viaje (Tv) & $-0,581(-3,952)$ & $-0,551(-3,980)$ \\
$($ C)to (C) & $-0,418(-2,644)$ & $-0,327(-2,713)$ \\
Frecuencias (Fr) & $0,016(2,762)$ & $0,015(2,801)$ \\
Seguridad_Terrestre (SEGT) & & $0,424(1,981)$ \\
Seguridad_Aéreo SEGA & & $0,872(2,542)$ \\
Comodidad_Terrestre COMT & & $0,342(2,015)$ \\
Comodidad_Aéreo COMA & $1,330(12,764)$ & $0,717(3,051)$ \\
Efecto de panel & $-3263,335$ & $1,160(12,271)$ \\
Log-verosimilitud & 0,248 & $-3103,371$ \\
$\rho^{2}$ & & 0,269 \\
\hline
\end{tabular}

Fuente: elaboración propia.

Respecto a las VLs, se puede observar que los efectos de la seguridad y la comodidad en la elección fueron estadísticamente significativos en el modelo híbrido. Como se observa en la Tabla 6, las percepciones de seguridad y comodidad de los viajeros tienen parámetros positivos para cada modo de transporte, esto implica que la utilidad del modo terrestre y aéreo aumenta con el nivel de seguridad y comodidad.

En lo relativo a la variable de políticas, el modelo híbrido muestra que los parámetros de las VLs seguridad y comodidad para los dos modos de transporte son altos en magnitud, comparados con el costo y las frecuencias. Esto implica que en este estudio las VLs dominan las otras variables, lo cual soporta la hipótesis que las percepciones de seguridad y comodidad 
Muñoz, Pineda Uribe y Gómez: Modelo econométrico para el análisis de la elección de modo...

están altamente asociadas con el comportamiento de elección de los viajeros. Además, con la inclusión de las VLs se mejora significativamente el ajuste del modelo. Lo anterior indica que evaluar las VLs seguridad y comodidad dentro de un modelo de elección permite una mejor estimación del proceso de elección para viajes domésticos. Al incluir las VLs dentro del modelo de elección también se puede observar que la constante específica reduce su importancia y su significancia estadística debido a que las VLs capturan factores intangibles en la elección de modo.

\section{Análisis predictivo}

El modelo econométrico estimado permitió calcular las cuotas de mercado de los modos de transporte considerados, es decir, la probabilidad de que el bus o el modo aéreo sean elegidos para realizar el trayecto MedellínBarranquilla. Para alimentar el modelo, se tomaron datos promedio anuales de los viajes realizados en el trayecto Medellín-Barranquilla en cada uno de los modos.

El modelo MNL asume que los errores son independientes e idénticamente distribuidos siguiendo una distribución Gumbel, con media cero y escala uno, lo cual implica una varianza de $\pi^{2} / 6$. (McFadden \& Domencich, 1975). Bajo estos supuestos la probabilidad de viajar en bus o en avión está dada por la siguiente ecuación:

$$
P_{i q}=\frac{\exp \left(V_{i q}\right)}{\sum_{j=1}^{J} \exp \left(V_{j q}\right)}
$$

En la Tabla 7 se indican los resultados de las cuotas de mercado para los modos estudiados en el trayecto Medellín-Barranquilla y se tiene que, para los valores de las variables presentados, la probabilidad de elección del modo aéreo es del 62,8\% y para el modo terrestre del 37,28\%. Esta diferencia se atribuye a la competencia de precios de las aerolíneas con el transporte terrestre, es decir, que para algunos usuarios del modo aéreo no les es difícil pagar una tarifa superior comparada con el terrestre, siempre y cuando esto represente un ahorro en tiempo de viaje, esto puede estar relacionado con 
el motivo del viaje, debido a que la mayoría de estos son por trabajo, lo cual indica que es más representativo el tiempo de viaje que el costo del tiquete.

Tabla 7. Probabilidad de elección del modo de transporte

\begin{tabular}{clccc}
\hline Modo & Variable & Valor & Utilidad & Probabilidad \\
\hline \multirow{4}{*}{ Aéreo } & Costo del Viaje (Ca) & 57 USD & $-31,722$ & $62,8 \%$ \\
& Tiempo Viaje (Tva) horas & 3 & & \\
& Frecuencias diarias (Fra) & 4 & & \\
\hline \multirow{3}{*}{ Terrestre } & Costo del Tiquete (Ct) & 50 USD & $-32,245$ & $37,2 \%$ \\
& Tiempo Viaje (Tvt) horas. & 15 & & \\
& Frecuencias diarias (Frt) & 14 & & \\
\hline
\end{tabular}

Fuente: elaboración propia.

\section{Conclusiones}

En el desarrollo de este artículo se aplicó la técnica de los grupos focales con el objetivo de encontrar patrones en el comportamiento de los viajeros. Con base en ellos, se determinaron las variables relevantes en la elección y los niveles de estas para construir modelos de demanda. Se identificaron cuatro tipos de variables a ser incluidas en los modelos de elección discreta; estas variables cuantitativas corresponden a tiempo de viaje, costo del viaje y la frecuencia de los vuelos; además de las variables latentes de seguridad y comodidad.

Las variables latentes fueron modeladas a partir de indicadores que recogen las percepciones de los pasajeros que integraron la muestra de estudio, encontrando como representativa la variable latente comodidad, su percepción en cuanto a la cercanía y facilidad para acceder al modo de transporte desde el origen, la suavidad en el desplazamiento y la del confort en cuanto a las sillas. En lo relacionado con la variable latente seguridad, se encontró que los indicadores de percepción en cuanto a la probabilidad de robo o atraco en el modo y el estado del equipaje al momento de la llegada permiten que esta pueda ser observada. 
Muñoz, Pineda Uribe y Gómez: Modelo econométrico para el análisis de la elección de modo...

Las ecuaciones de utilidad que permitieron calcular la probabilidad en la elección de un modo de transporte proporcionan elementos para la definición de políticas que promuevan el estudio de los efectos de la elección a causa de cambios en los atributos de los modos. Los modelos incluyeron variables más completas, las cuales permiten precisar implicaciones de gestión de un sistema de transporte.

Los modelos de elección discreta estimados a partir de datos de PD — tanto para usuarios del modo aéreo como del terrestre - mostraron que las variables tiempo — la cual incluye tiempo de acceso, de viaje y de espera-, costo - la cual está compuesta por la tarifa para cada uno de los modosy frecuencia resultaron ser significativas a la hora de elegir el modo de transporte, corroborando la percepción obtenida en el grupo focal.

Modelos más avanzados como los híbridos, permitieron introducir dentro de la función de utilidad variables latentes como la seguridad y la comodidad. Estas no son medibles directamente, sino que son explicadas por variables exógenas como lo son las características socioeconómicas — por ejemplo, sexo o edad-, y observadas a través de unos indicadores. Así, esta investigación contribuye con la literatura al introducir las variables latentes seguridad y comodidad dentro de la elección de un modo de transporte — bus o aviónpara un trayecto doméstico. Dichas variables resultaron significativas, lo que demuestra la importancia de incluir atributos de percepción para mejorar la capacidad predictiva de los modelos de elección.

\section{Referencias}

Aeronáutica Civil de Colombia. Unidad Administrativa Especial. (2017). La Aviación en cifras. Aeronáutica Civil de Colombia.

Ben-Akiva, M., Walker, J., Bernardino, A., Gopinath, D., \& Polydoropoulou, A. (2002a). Integration of Choice and Latent Variable Models. Massachusetts Institute of Technology.

Ben-Akiva, M., Walker, J., Bernardino, A., Gopinath, D., Morikawa, A., \& Polydoropoulou, A. (2002b). Hybrid Choice Models: Progress and 
challenges. Marketing Letters, 13, 163-175. https://doi.org/10.1023/A: 1020254301302

Bolduc, D., Boucher, N., \& Alvarez-Daziano, R. (2008). Hybrid Choice Modeling of New Technologies for Car Use in Canada. Transportation Research Record: Journal of the Transportation Research Board, 2082(1), 63-71. https://doi.org/10.3141/2082-08

Bollen, K. A. (1989). Structural Equations with Latent Variables. John Wiley and Sons.

Cantillo, V., Ortúzar, J. d., \& Williams, H. (2007). Modeling Discrete Choices in the Presence of Inertia and Serial Correlation. Transportation Science, 41(2), 195-205. https://doi.org/10.1287/trsc.1060.0178

Cochran, W. G. (1977). Sampling techniques. Third edition. John Wiley \& Sons.

Córdoba, J. E. (2010). Modelo de elección discreta integrando variables latentes y racionalidad limitada [tesis de doctorado, Universidad Nacional de Colombia]. https://repositorio.unal.edu.co/handle/unal/7636

Drabas, T., \& Wu, C. L. (2013). Modelling Air Carrier Choices with a Segment Specific Cross Nested Logit model. Journal of Air Transport Management, 32, 8-16. https://doi.org/10.1016/j.jairtraman.2013.04 .004

Espino, R., Ortúzar, J. d., \& Román García, C. (2004). Diseño de preferencias declaradas para analizar la demanda de viajes. Estudios de Economía Aplicada, 22(3), 759-793. https://www.redalyc.org/pdf/301/3012 2316.pdf

Fleischer, A., Tchetchik, A., \& Toledo, T. (2012). The Impact of Fear of Flying on Travelers' Flight Choice: Choice Model with Latent Variables. Journal of Travel Research, 51(5), 653-663. https://doi.org/10.1177/00 47287512437856

Hensher, D. A., Rose, J. M. and Greene, W. H. (2005). Applied Choice Analysis. A Primer. Cambridge University Press. 
Muñoz, Pineda Uribe y Gómez: Modelo econométrico para el análisis de la elección de modo...

Hyun, D. J., Ji, H. S., Doo, R. P., \& Hong, J. H. (2011). Inactivation of Glycogen Synthase Kinase-3B Is Required for Asteoclast Differentiation. Journal of Biological Chemistry, 286(45), 39043-39050. https: //doi.org/10.1074/jbc.M111.256768

Johansson, M., Heldt, T., \& Johansson, P. (2005). Latent Variables in a Travel Mode Choice Model: Attitudinal and Behavioral Indicator Variables [working paper No. 2005:5]. Department of Economics, Uppsala University. https://uu.diva-portal.org/smash/get/diva2:665210/F ULLTEXT01.pdf

Kim, J., Rasouli, S., \& Timmermans, H. (2017). Satisfaction and Uncertainty in Car-Sharing Decisions: An Integration of Hybrid Choice and Random Regret-Based Models. Transportation Research Part A: Policy and Practice, 95, 13-33. https://doi.org/10.1016/j.tra.2016.11.005

Kitrinou, E., Polydoropoulou, A., \& Bolduc, D. (2010). Development of Integrated Choice and Latent Variable (ICLV) Models for the Residential Relocation Decision in Island Areas. En S. Hess \& A. Daly (eds), Choice Modelling: The State-of-the-art and The State-of-practice (p.p. 593-618). Emerald.

Lee, T. T.-H., \& Yip, T. L. (2017). Impact of Flight Departure Delay on Airline Choice Behavior. Asian Journal of Shipping and Logistics, 33(4), 273-278. https://doi.org/10.1016/j.ajsl.2017.12.011

Márquez, L. (2016). La percepción de seguridad en la demanda de transporte de la integración bicicleta-metro en Bogotá, Colombia. Lecturas de Economía, 84, 143-177. https://doi.org/10.17533/udea.le.n84a05

Márquez, L., Macea, L. F. and Soto, J. J. (2019). Willingness to Change Car Use to Commute to the UPTC Main Campus, Colombia: A Hybrid Discrete Choice Modeling Approach. The Journal of Transport and Land Use, 12(1), 335-353. https://hdl.handle.net/20.500.12585/9238

McFadden, T. A., \& Domencich, D. (1975). Urban Travel Demand. A Behavioral Analysis. North-Holland Publishing Co. 
Morikawa, T., Ben-Akiva, M., \&. McFadden, D. (2002). Discrete Choice Models Incorporating Revealed Preferences and Psychometric Data. Advances in Econometrics, 16, 29-55. https://doi.org/10.1016/S073 1-9053(02)16003-8

Ortúzar, J. d., \& Willumsen, L. (2011). Modelling Transport. John Wiley \& Sons.

Szolnoki, G., \& Hoffmann, D. (2013). Online, Face-to-Face and Telephone Surveys - Comparing Different Sampling Methods in Wine Consumer Research. Wine Economics and Policy, 2(2), 57-66. https://doi.org/10.1 016/j.wep.2013.10.001

Tam, M., Lam, W., \& Lo, H. (2011). The Impact of Travel Time Reliability and Perceived Service Quality on Airport Ground Access Mode Choice. Journal of Choice Modelling, 4(2,), 49-69. https://doi.org/10.1016/S1 755-5345(13)70057-5

Terminales de Transporte Medellín S. A (2018). Movimientos de pasajeros 2017. Sociedad Terminales de Transporte Medellín.

Tsai, T., \& Chen, C. (2019). Mixed Logit Analysis of Trade-Off Effects between International Airline Fares and Fences: A Revenue Management Perspective. Current Issues in Tourism, 22(3), 265-275. https: //doi.org/10.1080/13683500.2017.1402869

Wen, C.-H., Wu, W.-N., \& Fu, C. (2019). Preferences for Alternative Travel Arrangements in Case of Major Flight Delays: Evidence from Choice Experiments with Prospect Theory. Transport Policy, 83, 111-119. https: //doi.org/10.1016/j.tranpol.2017.02.005

Yang, C.-W., Lu, J.-L. \& Hsu, C.-Y. (2014). Modeling Joint Airport and Route Choice Behavior for International and Metropolitan Airports. Journal of Air Transport Management, 39, 89-95. https://doi.org/10 .1016/j.jairtraman.2014.05.001

Yazdanpanah, M., \& Hosseinlou, M. (2016). The Influence of Personality Traits on Airport Public Transport Access Mode Choice: A Hybrid 
Muñoz, Pineda Uribe y Gómez: Modelo econométrico para el análisis de la elección de modo...

Latent Class Choice Modeling Approach. Journal of Air Transport Management, 55, 147-163. https://doi.org/10.1016/j.jairtraman.2 016.04 .010

Zochowska, R., Karoń, G., Sobota, A., \& Janecki, R. (2017). Selected Aspects of the Methodology of a Household Interview Survey on an Urban Agglomeration Scale with Regard to its Services. Scientific Journal of Silesian University of Technology. Series Transport, 95, 239-249. https: //doi.org/10.20858/sjsutst.2017.95.22 